\title{
有理圆雉曲线段的参数的几何意义 *
}

\author{
胡倩倩 王国瑾 ${ }^{* *}$ \\ (浙江大学数学系, 浙江大学 CAD\&CG 国家重点实验室, 杭州 310027)
}

摘要用代数和几何方法, 得到用有理二次或有理三次 Bézier 曲线表示的圆锥 曲线上的点与其参数域上的点所对应的函数关系; 即给出了有理圆锥曲线段的 表达式所描述的映射的逆映射公式. 这种公式用圆锥曲线段上此点和控制顶点 所决定的三角形面积、角度及有理 Bézier 曲线的权因子来表示, 或用此点和曲线 段首末端点相应的参数角度及有理 Bézier 曲线的权因子来表示. 这些结果对有 理 Bézier 曲线曲面的最佳参数化和重新参数化等算法实现是极其有益的.

\section{关键词 有理 Bézier 曲线 圆锥曲线段 椭圆 双曲线 抛物线 参数化}

\section{1 引言}

几何外形设计及机器制造中, 往往要求构造和表示由圆锥曲线段、直线段 和自由型曲线所组成的组合曲线形状, 因而圆弧曲线和圆雉曲线在造型系统中 占有重要的地位, 它们是形体表示和机械零件构图中简单而常用的元素之一. 国际上早期的 CAGD 系统中, 圆弧和圆雉曲线的数学表示形式是隐函数、几何 样条或 Ball 曲线等. 自从引进了 NURBS 技术以后, 人们利用有理 Bézier 曲线既 能表示多项式曲线 (如飞机机身上的纵向曲线), 又能表示圆雉曲线 (如飞机机身 上的横截面线) 的优点, 把它作为曲线设计系统的核心模型. 有关圆雉曲线的低 次有理 Bézier 表示的论著随之大量涌现 ${ }^{[1 \sim 10]}$.

如所周知, 当控制顶点相同时, 同一段圆雉曲线的有理 Bézier 表示不惟一, 原因在于不同的表示形式中, 圆雉曲线段上的点与参数域内的点的对应关系不 同, 即参数化方法不同. 为了选择圆雉曲线段的最佳设计参数, 彻底地搞清楚这 种对应关系的几何内在本质是十分重要的. 此外, 在有理 Bézier 曲线的离散、有

* 国家重点基础研究发展规划 (批准号: 2004CB719400)、国家自然科学基金 (批准号: 60373033, 60333010) 和国家自然科学基金创新研究群体 (批准号: 60021201) 资助项目

**E-mail: wgj@math.zju.edu.cn 
理 Bézier 曲面的几何连续等算法中也必须利用这种对应关系. 不但如此, 由于圆 雉曲线常作为骨架曲线用来构造调配曲面, 人们往往需要寻求改变其参数化方 式的正确途径和方法. 然而时至今日, 只有施法中 ${ }^{[10,11]}$ 找到了有理二次圆雉曲 线段上的点所对应的参数的一种几何意义, 以及用标准型有理二次 Bézier 曲线 表示圆弧时，圆弧上的点与参数之间用圆弧圆心角来表示的函数关系. 至于用 有理二次或有理三次 Bézier 曲线表示椭圆弧、双曲线弧及抛物线弧时，曲线上 的点与其参数域上的点所对应的函数关系及其内在本质至今还未加以充分的研 究. 本文正是在揭示有理二次圆雉曲线段上的点所对应的参数的另外 3 种几何 意义的基础上, 利用三角、几何原理和有理三次 Bézier 曲线表示圆雉曲线段的充

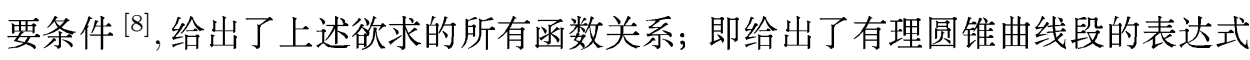
所描述的映射的逆映射公式. 这种公式用圆雉曲线段上此点和控制顶点所决定 的三角形面积、角度及有理 Bézier 曲线的权因子来表示, 或用此点和曲线段首末 端点相应的参数角度及有理 Bézier 曲线的权因子来表示. 这些结果对有理 Bézier 曲线曲面的最佳参数化及重新参数化等算法的实现无疑是十分有益的.

\section{2 有理二次圆雉曲线段上的点与其参数域上的点之间的关系}

\section{1 有理二次圆雉曲线段}

圆雉曲线段的有理二次 Bézier 表示形式为 (见图 1)

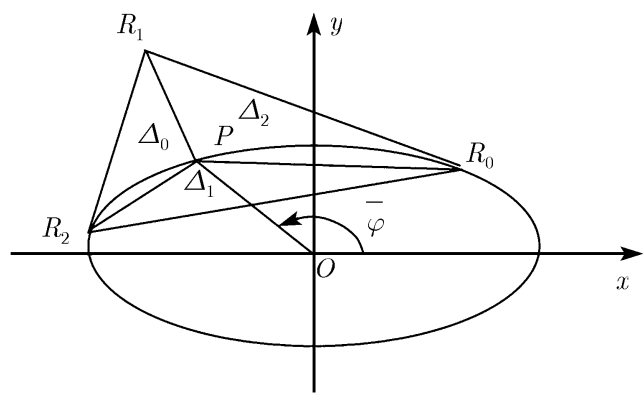

图 1 用有理二次 Bézier 曲线表示的圆雉曲线段及曲线上一点 $P$

$$
R(t)=\frac{\sum_{i=0}^{2} B_{i}^{2}(t) \omega_{i} R_{i}}{\sum_{i=0}^{2} B_{i}^{2}(t) \omega_{i}}, \quad 0 \leqslant t \leqslant 1,
$$

其中 $R(t)$ 表示对应于参数 $t$ 的圆雉曲线段上的点; $R_{i}=\left(x_{i}, y_{i}\right)(i=0,1,2)$ 为 3 个控制顶点; $\omega_{i}>0(i=0,1,2)$ 为 3 个权因子; $B_{i}^{2}(t)=\left(\begin{array}{c}2 \\ i\end{array}\right)(1-t)^{2-i} t^{i}$ 为二次 Bernstein 基函数.

首先我们给出平面上一点位于有理二次 Bézier 曲线上的充要条件, 然后给 出曲线上任一点所对应的参数的几种几何意义. 不失一般性, 假设 $R_{0}, R_{1}, R_{2}$ 三点不共线, 否则曲线 $R(t)$ 就是一条退化的圆雉曲线, 即一条线段. 
定理 1 假设点 $P$ 为 $\triangle R_{0} R_{1} R_{2}$ 内的任一点, $\Delta_{0}, \Delta_{1}, \Delta_{2}$ 分别是 $\triangle P R_{1} R_{2}$, $\triangle R_{0} P R_{2}, \triangle R_{0} R_{1} P$ 的有向面积, 则点 $P$ 落在有理二次 Bézier 曲线 (1) 上, 当且 仅当下式成立:

$$
\frac{\Delta_{1}^{2}}{4 \Delta_{0} \Delta_{2}}=\frac{\omega_{1}^{2}}{\omega_{0} \omega_{2}} .
$$

证 记 $\triangle R_{0} R_{1} R_{2}$ 的有向面积为 $\Delta$. 若点 $P$ 落在曲线 (1) 上, 则存在一个实 数 $t \in[0,1]$, 使得

$$
P=\frac{(1-t)^{2} \omega_{0}}{\sum_{i=0}^{2} B_{i}^{2}(t) \omega_{i}} R_{0}+\frac{2(1-t) t \omega_{1}}{\sum_{i=0}^{2} B_{i}^{2}(t) \omega_{i}} R_{1}+\frac{t^{2} \omega_{2}}{\sum_{i=0}^{2} B_{i}^{2}(t) \omega_{i}} R_{2} .
$$

按重心坐标的定义, 上式意味着

$$
\frac{1}{\Delta}\left(\begin{array}{c}
\Delta_{0} \\
\Delta_{1} \\
\Delta_{2}
\end{array}\right)=\left(\frac{(1-t)^{2} \omega_{0}}{\sum_{i=0}^{2} B_{i}^{2}(t) \omega_{i}}, \frac{2(1-t) t \omega_{1}}{\sum_{i=0}^{2} B_{i}^{2}(t) \omega_{i}}, \frac{t^{2} \omega_{2}}{\sum_{i=0}^{2} B_{i}^{2}(t) \omega_{i}}\right)^{\mathrm{T}} .
$$

于是 (2) 式成立. 反之, 若 (2) 式成立, 则 $\left(\omega_{0} \Delta_{1}\right)^{2} \omega_{2}=4 \omega_{0} \omega_{1}^{2} \Delta_{0} \Delta_{2}$. 利用上式中 $\Delta_{0}$ 与 $\Delta_{1}$ 的比值, 取参数 $t=\frac{\omega_{0} \Delta_{1}}{\omega_{0} \Delta_{1}+2 \omega_{1} \Delta_{0}} \in[0,1]$, 就有

$$
\left((1-t)^{2} \omega_{0}, 2(1-t) t \omega_{1}, t^{2} \omega_{2}\right)^{\mathrm{T}}=\frac{4 \omega_{0} \omega_{1}^{2} \Delta_{0}}{\left(\omega_{0} \Delta_{1}+2 \omega_{1} \Delta_{0}\right)^{2}}\left(\begin{array}{c}
\Delta_{0} \\
\Delta_{1} \\
\Delta_{2}
\end{array}\right),
$$

所以 $R(t)=\Delta^{-1}\left(\Delta_{0} R_{0}+\Delta_{1} R_{1}+\Delta_{2} R_{2}\right)=P$, 即点 $P$ 落在曲线 (1) 上. 证毕.

定理 2 假设点 $P$ 落在有理二次 Bézier 曲线 (1) 上, 则它所对应的参数必为

$$
t=\frac{\omega_{0} \Delta_{1}}{\omega_{0} \Delta_{1}+2 \omega_{1} \Delta_{0}}=\frac{2 \omega_{1} \Delta_{2}}{2 \omega_{1} \Delta_{2}+\omega_{2} \Delta_{1}}=\frac{\sqrt{\omega_{0} \Delta_{2}}}{\sqrt{\omega_{0} \Delta_{2}}+\sqrt{\omega_{2} \Delta_{0}}},
$$

这里 $\Delta_{0}, \Delta_{1}, \Delta_{2}$ 的意义同定理 1 .

证 由定理 1 可知 $R\left(\frac{\omega_{0} \Delta_{1}}{\omega_{0} \Delta_{1}+2 \omega_{1} \Delta_{0}}\right)=P$, 又因有理二次 Bézier 曲线无重点, 所以点 $P$ 和参数 $t=\frac{\omega_{0} \Delta_{1}}{\omega_{0} \Delta_{1}+2 \omega_{1} \Delta_{0}}$ 存在着一一对应的关系. 其次把 (3) 式改写为

$$
t=\frac{1}{1+2 \frac{\omega_{1}}{\omega_{0}} \frac{\Delta_{0}}{\Delta_{1}}}=\frac{1}{1+\frac{1}{2} \frac{\omega_{2}}{\omega_{1}} \frac{\Delta_{1}}{\Delta_{2}}}=\frac{1}{1+\sqrt{\frac{\omega_{2}}{\omega_{0}} \frac{\Delta_{0}}{\Delta_{2}}}} .
$$

而根据 (2) 式容易得到

$$
2 \frac{\omega_{1}}{\omega_{0}} \frac{\Delta_{0}}{\Delta_{1}}=\frac{1}{2} \frac{\omega_{2}}{\omega_{1}} \frac{\Delta_{1}}{\Delta_{2}}=\sqrt{\frac{\omega_{2}}{\omega_{0}} \frac{\Delta_{0}}{\Delta_{2}}} .
$$

于是 (4) 式成立, 从而 (3) 式成立. 证毕.

必须指出, 上述 (3) 式中的第 3 式是由施法中 [10] 首先给出的. 但他的证明 要应用 Faux ${ }^{[1]}$ 等人的结果, 我们的证明可由定理 1 直接得到, 且可以同时得出 (3) 式中的其他两个公式.

定理 3 假设点 $P$ 落在有理二次 Bézier 曲线 (1) 上, 则它所对应的参数必为

$$
t=\frac{1}{1+\sqrt{\frac{\omega_{2}}{\omega_{0}}\left(\frac{\cot \beta-\cot C}{\cot \alpha-\cot A}\right)}},
$$


这里 $\alpha=\angle P R_{0} R_{2}, \beta=\angle P R_{2} R_{0}, A=\angle R_{1} R_{0} R_{2}, C=\angle R_{1} R_{2} R_{0}$ (见图 2).

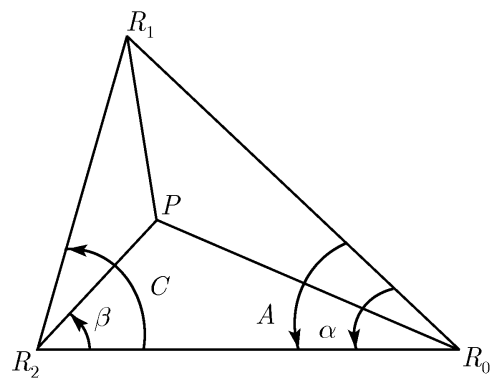

图 2 有理圆雉曲线上的点和控制顶点的连线生成的三角形

证 由正弦定理, 可得

$$
\frac{P R_{2}}{P R_{0}}=\frac{\sin \alpha}{\sin \beta}, \quad \frac{R_{1} R_{2}}{R_{0} R_{1}}=\frac{\sin A}{\sin C},
$$

因而

$$
\begin{aligned}
\frac{\Delta_{0}}{\Delta_{2}} & =\frac{\operatorname{area}\left(\triangle P R_{1} R_{2}\right)}{\operatorname{area}\left(\triangle R_{0} R_{1} P\right)}=\frac{\left(R_{1} P \cdot \sin \angle R_{1} P R_{2}\right) \cdot P R_{2}}{\left(R_{1} P \cdot \sin \angle R_{1} P R_{0}\right) \cdot P R_{0}} \\
& =\frac{\left(R_{1} R_{2} \cdot \sin \angle R_{1} R_{2} P\right)}{\left(R_{0} R_{1} \cdot \sin \angle R_{1} R_{0} P\right)} \cdot \frac{\sin \alpha}{\sin \beta} \\
& =\frac{\sin A}{\sin C} \cdot \frac{\sin (C-\beta)}{\sin (A-\alpha)} \cdot \frac{\sin \alpha}{\sin \beta}=\frac{\cot \beta-\cot C}{\cot \alpha-\cot A} .
\end{aligned}
$$

于是利用 (4) 式中的第 3 式得到 (5) 式, 证毕.

定理 2 和 3 实质上给出了有理二次圆雉曲线段的表达式 (1) 所描述的映射 $t \in[0,1] \mapsto R \in \mathbb{R}^{2}$ 的逆映射 $R \in \mathbb{R}^{2} \mapsto t \in[0,1]$ 的公式. 即按表达式 (1), 由每一 个给定的参数值 $t$, 我们能决定曲线上点的位置; 反之, 按表达式 (3) 或 (5), 由曲 线上每一个给定的点, 我们能决定参数值 $t$. 影响参数值 $t$ 的量既有曲线段的权 因子, 又有由曲线段的控制顶点和此点所决定的三角形面积、角度等几何量. 下 面分别讨论曲线为椭圆弧、双曲线弧和抛物线弧时, 其参数的几何意义. 分析表 明，这个参数值能由曲线段的权因子以及指定点和曲线段首末端点的参数角惟 一决定. 定义位置角如下:

定义 1 设点 $P$ 位于圆雉曲线上, 点 $O$ 为坐标系的原点, 则由正 $x$ 轴到射 线 $O P$ 的有向转角称为点 $P$ 在圆雉曲线上的位置角 (见图 1).

\section{2 有理二次椭圆弧、双曲线弧和抛物线弧}

首先考察有理二次椭圆弧. 由有理 Bézier 曲线的几何不变性, 不妨以椭圆 的中心为原点, 以椭圆的长轴和短轴为 $x$ 和 $y$ 轴建立直角坐标系. 设此椭圆的 方程为 $\frac{x^{2}}{a^{2}}+\frac{y^{2}}{b^{2}}=1(a \geqslant b>0)$, 再设曲线段上的点和首末端点相应的位置角分 别为 $\bar{\theta}=\bar{\varphi}, \bar{\xi}, \bar{\eta}$ (见图 3), 其相应的参数角分别为 $\theta=\varphi, \xi, \eta$. 它们满足关系式 $\tan \theta=\frac{b}{a} \tan \bar{\theta}$, 故此椭圆弧的参数方程可表示为 


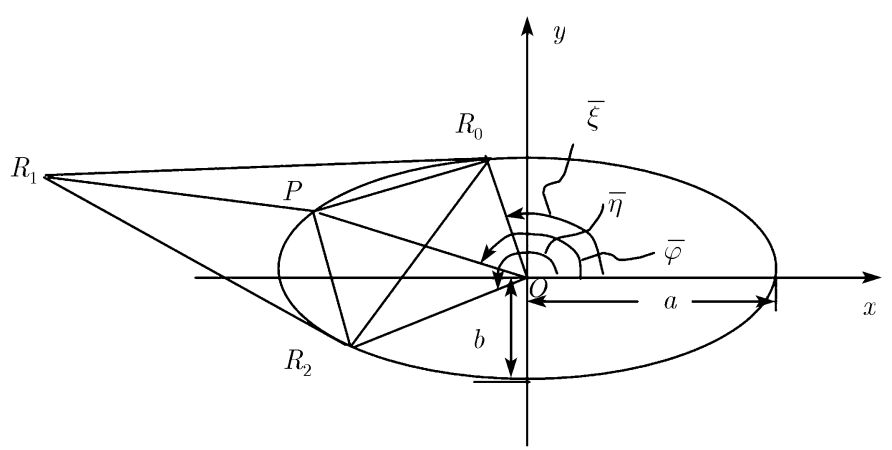

图 3 用有理二次 Bézier 曲线表示的椭圆弧

$$
f_{1}(\varphi)=\left(\begin{array}{c}
a \cos \varphi \\
b \sin \varphi
\end{array}\right), \quad 0 \leqslant \xi \leqslant \varphi \leqslant \eta \leqslant 2 \pi .
$$

此时，椭圆弧在端点处有

$$
\begin{aligned}
& R^{\prime}(0)=\left.\left.f_{1}^{\prime}\right|_{\varphi=\xi} \cdot \frac{d \varphi}{d t}\right|_{t=0}=\left.\left(\begin{array}{c}
-a \sin \xi \\
b \cos \xi
\end{array}\right) \cdot \frac{d \varphi}{d t}\right|_{t=0}, \\
& R^{\prime}(1)=\left.\left.f_{1}^{\prime}\right|_{\varphi=\eta} \cdot \frac{d \varphi}{d t}\right|_{t=1}=\left.\left(\begin{array}{c}
-a \sin \eta \\
b \cos \eta
\end{array}\right) \cdot \frac{d \varphi}{d t}\right|_{t=1} .
\end{aligned}
$$

按照有理二次 Bézier 曲线的端点导矢公式 [9] 可得

$$
R_{1}=\left(\begin{array}{c}
a \cos \xi-\left.\frac{a \omega_{0}}{2 \omega_{1}} \cdot \frac{d \varphi}{d t}\right|_{t=0} \cdot \sin \xi \\
b \sin \xi+\left.\frac{b \omega_{0}}{2 \omega_{1}} \cdot \frac{d \varphi}{d t}\right|_{t=0} \cdot \cos \xi
\end{array}\right)=\left(\begin{array}{c}
a \cos \eta+\left.\frac{a \omega_{2}}{2 \omega_{1}} \cdot \frac{d \varphi}{d t}\right|_{t=1} \cdot \sin \eta \\
b \sin \eta-\left.\frac{b \omega_{2}}{2 \omega_{1}} \cdot \frac{d \varphi}{d t}\right|_{t=1} \cdot \cos \eta
\end{array}\right) .
$$

今以 $k(M N)$ 表示直线 $M N$ 之斜率, 容易算出

$$
\begin{gathered}
k\left(R_{0} P\right)=-\frac{b}{a} \cot \frac{\xi+\varphi}{2}, \quad k\left(R_{0} R_{2}\right)=-\frac{b}{a} \cot \frac{\xi+\eta}{2}, \\
k\left(P R_{2}\right)=-\frac{b}{a} \cot \frac{\varphi+\eta}{2}, \\
k\left(R_{0} R_{1}\right)=-\frac{b}{a} \cot \xi, \quad k\left(R_{1} R_{2}\right)=-\frac{b}{a} \cot \eta,
\end{gathered}
$$

故

$$
\begin{aligned}
& \tan \alpha= \frac{k\left(R_{0} R_{2}\right)-k\left(R_{0} P\right)}{1+k\left(R_{0} R_{2}\right) \cdot k\left(R_{0} P\right)}=\frac{a b \sin \frac{\eta-\varphi}{2}}{a^{2} \sin \frac{\xi+\eta}{2} \sin \frac{\xi+\varphi}{2}+b^{2} \cos \frac{\xi+\varphi}{2} \cos \frac{\xi+\eta}{2}}, \\
& \tan A=\frac{k\left(R_{0} R_{2}\right)-k\left(R_{0} R_{1}\right)}{1+k\left(R_{0} R_{2}\right) \cdot k\left(R_{0} R_{1}\right)}=\frac{a b \sin \frac{\eta-\xi}{2}}{a^{2} \sin \xi \sin \frac{\xi+\eta}{2}+b^{2} \cos \xi \cos \frac{\xi+\eta}{2}},
\end{aligned}
$$

且

$$
\tan \beta=\frac{k\left(P R_{2}\right)-k\left(R_{0} R_{2}\right)}{1+k\left(P R_{2}\right) \cdot k\left(R_{0} R_{2}\right)}, \quad \tan C=\frac{k\left(R_{1} R_{2}\right)-k\left(R_{0} R_{2}\right)}{1+k\left(R_{1} R_{2}\right) \cdot k\left(R_{0} R_{2}\right)}
$$


可用同样的方法算出. 根据以上 4 式, 并注意到

$$
\begin{aligned}
& \sin \frac{\eta-\xi}{2} \sin \frac{\varphi+\eta}{2}-\sin \frac{\varphi-\xi}{2} \sin \eta \\
= & -\frac{1}{2}\left[\cos \left(\frac{\varphi-\xi}{2}+\eta\right)-\cos \left(\frac{\xi+\varphi}{2}\right)\right] \\
& +\frac{1}{2}\left[\cos \left(\frac{\varphi-\xi}{2}+\eta\right)-\cos \left(\frac{\varphi-\xi}{2}-\eta\right)\right] \\
= & \frac{1}{2}\left[\cos \frac{\xi+\varphi}{2}-\cos \left(\frac{\varphi-\xi}{2}-\eta\right)\right]=\sin \frac{\eta-\varphi}{2} \sin \frac{\xi+\eta}{2},
\end{aligned}
$$

故有

$$
\frac{\cot \beta-\cot C}{\cot \alpha-\cot A}=\frac{\sin ^{2} \frac{\eta-\varphi}{2}}{\sin ^{2} \frac{\varphi-\xi}{2}} .
$$

由此应用定理 3, 可得到曲线上点 $P=f_{1}(\varphi)(\xi \leqslant \varphi \leqslant \eta)$ 所对应的参数为

$$
t=t(\varphi)=\frac{\sin \frac{\varphi-\xi}{2}}{\sin \frac{\varphi-\xi}{2}+\sin \frac{\eta-\varphi}{2} \sqrt{\frac{\omega_{2}}{\omega_{0}}}},
$$

它关于 $\varphi$ 的反函数为

$$
\varphi=\varphi(t)=2 \arctan \frac{(1-t) \sin \frac{\xi}{2}+t \sin \frac{\eta}{2} \sqrt{\frac{\omega_{2}}{\omega_{0}}}}{(1-t) \cos \frac{\xi}{2}+t \cos \frac{\eta}{2} \sqrt{\frac{\omega_{2}}{\omega_{0}}}} .
$$

下面考察有理二次双曲线弧, 当曲线为双曲线 $\frac{x^{2}}{a^{2}}-\frac{y^{2}}{b^{2}}=1(a, b>0, x>0)$ 的一部分时, 令 $\bar{\theta}=\bar{\varphi}, \bar{\xi}, \bar{\eta}, \theta=\varphi, \xi, \eta$ 的意义与椭圆弧时类似 (见图 4), 则 $\bar{\theta}$ 与 $\theta$ 满足关系 $\tan \theta=\frac{b}{a} \sin \bar{\theta}$, 此双曲线弧的参数方程可表示为

$$
f_{2}(\varphi)=\left(\begin{array}{c}
a / \cos \varphi \\
b \tan \varphi
\end{array}\right), \quad-\arctan \frac{b}{a}<\xi \leqslant \varphi \leqslant \eta<\arctan \frac{b}{a} .
$$

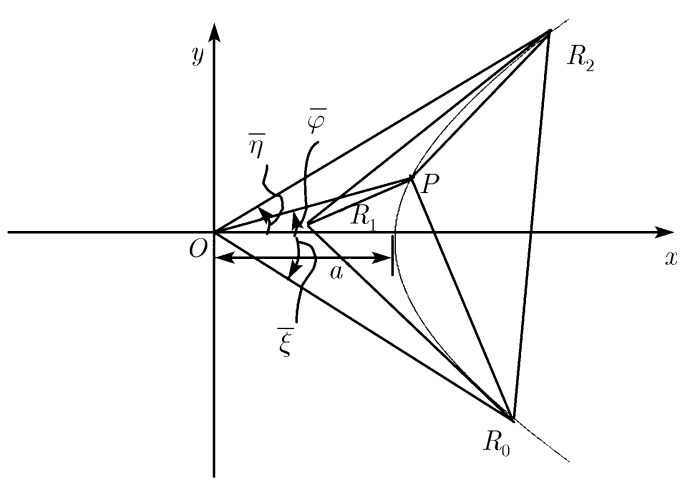

图 4 用有理二次 Bézier 曲线表示的双曲线弧 
用处理椭圆弧相同的方法, 可以求出曲线上点 $P=f_{2}(\varphi)(\xi \leqslant \varphi \leqslant \eta)$ 所对 应的参数为

$$
t=t(\varphi)=\frac{\sin \frac{\varphi-\xi}{2}}{\sin \frac{\varphi-\xi}{2}+\sin \frac{\eta-\varphi}{2} \sqrt{\frac{\omega_{2}}{\omega_{0}} \cdot \frac{\cos \xi}{\cos \eta}}} .
$$

而参数 $t$ 关于参数角 $\varphi$ 的反函数为

$$
\varphi=\varphi(t)=2 \arctan \frac{(1-t) \sin \frac{\xi}{2}+t \sin \frac{\eta}{2} \sqrt{\frac{\omega_{2}}{\omega_{0}} \cdot \frac{\cos \xi}{\cos \eta}}}{(1-t) \cos \frac{\xi}{2}+t \cos \frac{\eta}{2} \sqrt{\frac{\omega_{2}}{\omega_{0}} \cdot \frac{\cos \xi}{\cos \eta}}} .
$$

最后考察有理二次抛物线弧. 当曲线为抛物线 $x=\frac{1}{4 p} y^{2}-p(p>0)$ 的一部 分时, 令 $\bar{\theta}=\bar{\varphi}, \bar{\xi}, \bar{\eta}, \theta=\varphi, \xi, \eta$ 的意义与椭圆弧时类似 (见图 5), 则 $\bar{\theta}$ 与 $\theta$ 的关 系满足 $\bar{\theta}=\theta$. 此抛物线弧的参数方程可表示为

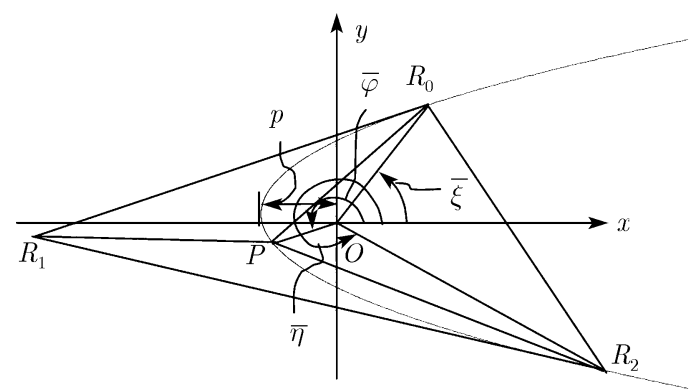

图 5 用有理二次 Bézier 曲线表示的抛物线弧

$$
f_{3}(\varphi)=\left(\begin{array}{c}
p\left(\cot ^{2} \frac{\varphi}{2}-1\right) \\
2 p \cot \frac{\varphi}{2}
\end{array}\right), \quad 0<\xi \leqslant \varphi \leqslant \eta<2 \pi .
$$

用处理椭圆弧同样的方法, 可以求出曲线上点 $P=f_{3}(\varphi)(\xi \leqslant \varphi \leqslant \eta)$ 所对应的参 数为

$$
t=t(\varphi)=\frac{\sin \frac{\varphi-\xi}{2} \sin \frac{\eta}{2}}{\sin \frac{\varphi-\xi}{2} \sin \frac{\eta}{2}+\sin \frac{\eta-\varphi}{2} \sin \frac{\xi}{2} \sqrt{\frac{\omega_{2}}{\omega_{0}}}} .
$$

而参数 $t$ 关于参数角 $\varphi$ 的反函数为

$$
\varphi=\varphi(t)=2 \arctan \frac{(1-t)+t \cdot \sqrt{\frac{\omega_{2}}{\omega_{0}}}}{(1-t) \cot \frac{\xi}{2}+t \cot \frac{\eta}{2} \cdot \sqrt{\frac{\omega_{2}}{\omega_{0}}}} .
$$

总结上述内容, 我们得到

定理 4 用有理二次 Bézier 曲线 (1) 表示由 (7)、(10)、(13) 式所给出的椭圆 弧、双曲线弧和抛物线弧时, 曲线上任一点 $P$ 所对应的参数的几何意义分别如 (8)、(11)、(14) 式所示, 或 (9)、(12)、(15) 式所示. 


\section{3 应用}

在曲线造型或曲线形式转换中，有时设计系统的用户对已知的有理二次 Bézier 曲线 (1) 上的某一点 $P$ 按照上述表达式 (3)、(5)、(8)、(11)、(14) 计算 出来的对应参数值 $t$ 并不满意, 而希望赋予另一个参数值 $u \in(0,1)$. 容易知道, 这可以在条件

$$
\omega_{0} \omega_{2} / \omega_{1}^{2}=\omega_{0}^{*} \omega_{2}^{*} / \omega_{1}^{* 2}
$$

之下，通过对曲线 (1) 引进一个分式线性参数变换

$$
t=\frac{u}{u+\sqrt{\frac{\omega_{0}^{*}}{\omega_{0}} / \frac{\omega_{2}^{*}}{\omega_{2}}}(1-u)}, \quad 0 \leqslant u \leqslant 1,
$$

把曲线 (1) 变换到参数为 $u$, 权因子为 $\omega_{i}^{*}(i=0,1,2)$, 但控制顶点和形状均不变的 有理二次 Bézier 曲线 $R^{*}(u)(0 \leqslant u \leqslant 1)$ 来实现. 其做法是: 首先把表达式 (3)、 (5)、(8)(或 (11)、(14)) 中的 $t$ 记为 $u, \omega_{i}$ 记为 $\omega_{i}^{*}(i=0,1,2)$, 由此解出比值 $\omega_{2}^{*} / \omega_{0}^{*}$, 或 $\omega_{2}^{*} / \omega_{1}^{*}$ 或 $\omega_{1}^{*} / \omega_{0}^{*}$; 再进一步应用条件 (16) 选择全部权因子 $\omega_{i}^{*}(i=0,1,2)$, 并得 到一个分式线性参数变换 (17) 式. 可见定理 $2 \sim 4$ 为有理二次 Bézier 曲线在用户 随意指定点处的重新参数化提供了算法的理论基础. 对曲线 (1) 的重新参数化, 一般有如下的

推论 1 对有理二次 Bézier 曲线 (1) 所表示的圆雉曲线段, 若保持 $\omega_{1}^{2} /\left(\omega_{0} \omega_{2}\right)$ 之值不变, 但调整权因子比值 $\omega_{0} / \omega_{2}$, 或 $\omega_{1} / \omega_{2}$, 或 $\omega_{0} / \omega_{1}$, 则当以上任一比值增大 (减小) 时, 曲线上点 $P$ 的原参数值 $t$ 将随之增大 (减小).

\section{3 有理三次圆雉曲线段上的点与其参数域上的点之间的关系}

圆雉曲线段的有理三次 Bézier 表示形式为

$$
R(t)=\frac{\sum_{i=0}^{3} B_{i}^{3}(t) \omega_{i} R_{i}}{\sum_{i=0}^{3} B_{i}^{3}(t) \omega_{i}}, \quad 0 \leqslant t \leqslant 1,
$$

其中 $R(t)$ 表示对应于参数 $t$ 的圆雉曲线段上的点; $R_{i}=\left(x_{i}, y_{i}\right)(i=0,1,2,3)$ 为 4 个控制顶点，任三点不共线; $\omega_{i}>0(i=0,1,2,3)$ 为 4 个权因子; $B_{i}^{3}(t)=$ $\left(\begin{array}{l}3 \\ i\end{array}\right)(1-t)^{3-i} t^{i}$ 为三次 Bernstein 基函数.

为了分析有理三次 Bézier 曲线所表示的圆雉曲线上每一点的参数的几何意 义, 首先需要引入下列两个引理. 引理的证明可见文献 [8]:

引理 1 有理三次 Bézier 曲线 (18) 是圆雉曲线段, 当且仅当方程组

$$
\left\{\begin{array}{l}
\omega_{0}-3 \omega_{1} k+3 \omega_{2} k^{2}-\omega_{3} k^{3}=0, \\
\omega_{0} R_{0}-3 \omega_{1} R_{1} k+3 \omega_{2} R_{2} k^{2}-\omega_{3} R_{3} k^{3}=0
\end{array}\right.
$$

有惟一的根

$$
k=\frac{\omega_{0}}{\omega_{1}} \cdot \frac{S_{1}}{3 S_{0}}=\frac{\omega_{1}}{\omega_{2}} \cdot \frac{S_{2}}{S_{1}}=\frac{\omega_{2}}{\omega_{3}} \cdot \frac{3 S_{3}}{S_{2}},
$$


这里 $S_{i}(i=0,1,2,3)$ 分别是 $\triangle R_{1} R_{2} R_{3}, \triangle R_{0} R_{2} R_{3}, \triangle R_{0} R_{1} R_{3}$ 和 $\triangle R_{0} R_{1} R_{2}$ 的 有向面积 (见图 6).
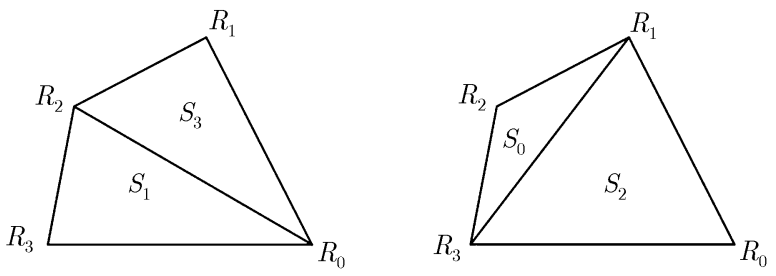

图 6 有向面积 $S_{0}, S_{1}, S_{2}, S_{3}$

引理 2 若有理三次 Bézier 曲线 (18) 是圆雉曲线段, 则可按照 (19) 式给出 的实数值 $k$, 作分式线性变换

$$
t=k u /(k u+(1-u)),
$$

把参数 $t$ 换作参数 $u$, 产生 1 条与曲线 (18) 等同的有理二次 Bézier 曲线 (见图 7)
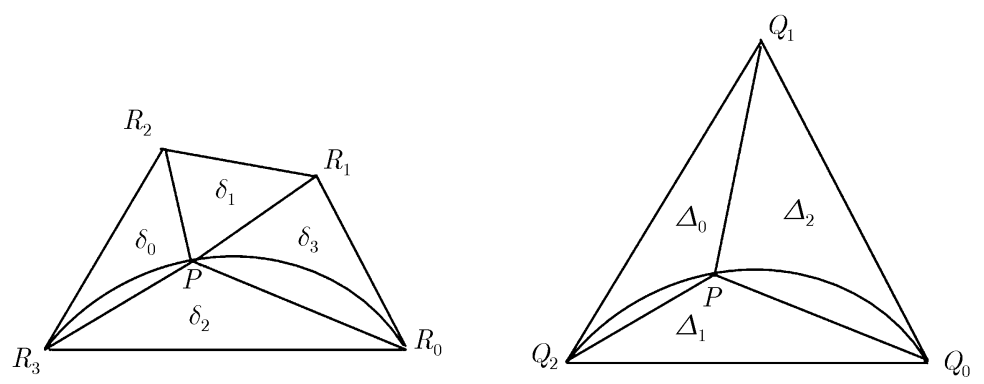

图 7 形状等同的两条有理圆雉曲线段 $R(t), \bar{R}(u)$ 及其控制多边形

$$
\bar{R}(u)=\frac{B_{0}^{2}(u) \lambda_{0} Q_{0}+B_{1}^{2}(u) \lambda_{1} Q_{1}+B_{2}^{2}(u) \lambda_{2} Q_{2}}{B_{0}^{2}(u) \lambda_{0}+B_{1}^{2}(u) \lambda_{1}+B_{2}^{2}(u) \lambda_{2}}, \quad 0 \leqslant u \leqslant 1,
$$

这里

$$
\begin{array}{r}
\left(\lambda_{0}, \lambda_{1}, \lambda_{2}\right)=\left(\omega_{0},\left(-\omega_{0}+3 \omega_{1} k\right) / 2=\left(-\omega_{3} k^{3}+3 \omega_{2} k^{2}\right) / 2, \omega_{3} k^{3}\right), \\
\left(Q_{0}, Q_{1}, Q_{2}\right)=\left(R_{0}, \frac{-\omega_{0} R_{0}+3 \omega_{1} k R_{1}}{-\omega_{0}+3 \omega_{1} k}=\frac{-\omega_{3} k^{3} R_{3}+3 \omega_{2} k^{2} R_{2}}{-\omega_{3} k^{3}+3 \omega_{2} k^{2}}, R_{3}\right) .
\end{array}
$$

根据引理 1 和 2 , 可得到

定理 5 设 $R(t)$ 和 $\bar{R}(u)$ 是由引理 2 给定的有理三次和有理二次圆雉曲线 段, $S_{i}(i=0,1,2,3)$ 是由引理 1 所给定的有向面积, 点 $P$ 在圆雉曲线段 $R(t)$ 上, $\Delta_{i}(i=0,1,2)$ 分别是 $\triangle P Q_{1} Q_{2}, \triangle Q_{0} P Q_{2}, \triangle Q_{0} Q_{1} P$ 的有向面积 (见图 7), $k$ 为 由 (19) 式决定的实数, 则点 $P$ 所对应的参数必为

$$
t=\frac{1}{1+\sqrt[6]{\left(\frac{\omega_{3}}{\omega_{0}}\right)^{2} \cdot \frac{S_{3}}{S_{0}}} \cdot \sqrt{\frac{\Delta_{0}}{\Delta_{2}}}}=\frac{1}{1+\sqrt{k \cdot \frac{\omega_{3}}{\omega_{0}} \cdot \frac{\Delta_{0}}{\Delta_{2}}}} .
$$


再设四边形 $R_{0} R_{1} R_{2} R_{3}$ 各个顶点与点 $P$ 的连线把此四边形所分割成的 4 个 三角形 $\left(\triangle P R_{2} R_{3}, \triangle R_{1} R_{2} P, \triangle R_{0} P R_{3}\right.$ 和 $\left.\triangle R_{0} R_{1} P\right)$ 的有向面积依次为 $\delta_{i}(i=$ $0,1,2,3)$ (见图 7), 则点 $P$ 所对应的参数必可表为

$$
t=\frac{1}{1+\sqrt{k^{2} \cdot \frac{\omega_{2} \omega_{3}}{\omega_{0} \omega_{1}} \cdot \frac{\delta_{0}}{\delta_{3}}}} .
$$

证 记 $\triangle Q_{0} Q_{1} Q_{2}$ 的有向面积为 $\Delta$. 由重心坐标的定义知

$$
P=\Delta^{-1}\left(\Delta_{0} Q_{0}+\Delta_{1} Q_{1}+\Delta_{2} Q_{2}\right) .
$$

应用表达式 (3) 的第 3 式和 (22) 式，立即知道 $P$ 点所对应的参数为

$$
u=\frac{1}{1+\sqrt{\frac{\lambda_{2}}{\lambda_{0}} \frac{\Delta_{0}}{\Delta_{2}}}}=\frac{1}{1+\sqrt{k^{3} \frac{\omega_{3}}{\omega_{0}} \frac{\Delta_{0}}{\Delta_{2}}}} .
$$

另一方面, 将 (23) 式代入 (26) 式, 有

$$
\begin{aligned}
P & =\frac{1}{\Delta}\left(\left(\Delta_{0}+\frac{-\omega_{0} \Delta_{1}}{-\omega_{0}+3 \omega_{1} k}\right) R_{0}+\frac{3 \omega_{1} k \Delta_{1}}{-\omega_{0}+3 \omega_{1} k} R_{1}+\Delta_{2} R_{3}\right) \\
& =\frac{1}{\Delta}\left(\Delta_{0} R_{0}+\frac{3 \omega_{2} k^{2} \Delta_{1}}{-\omega_{3} k^{3}+3 \omega_{2} k^{2}} R_{2}+\left(\frac{-\omega_{3} k^{3} \Delta_{1}}{-\omega_{3} k^{3}+3 \omega_{2} k^{2}}+\Delta_{2}\right) R_{3}\right) .
\end{aligned}
$$

于是由重心坐标的惟一性可得

$$
\Delta_{0} / \Delta=\delta_{0} / S_{1}, \quad \Delta_{2} / \Delta=\delta_{3} / S_{2} .
$$

又由 (19) 式可知

$$
\frac{S_{2}}{S_{1}}=k \cdot \frac{\omega_{2}}{\omega_{1}}, \quad k=\sqrt[3]{\frac{\omega_{0}}{\omega_{3}} \frac{S_{3}}{S_{0}}} .
$$

把以上 4 式代入 (27) 式并且应用 (22) 式, 可得

$$
u=\frac{1}{1+\sqrt{\frac{S_{3}}{S_{0}} \frac{\Delta_{0}}{\Delta_{2}}}}=\frac{1}{1+\sqrt{k^{4} \cdot \frac{\omega_{2} \omega_{3}}{\omega_{0} \omega_{1}} \cdot \frac{\delta_{0}}{\delta_{3}}}} .
$$

最后根据 (20) 式, 我们能求出由 (24) 和 (25) 式所给的 $t$ 值. 证毕.

定理 5 实质上给出了有理三次圆雉曲线段的表达式 (18) 所描述的映射 $t \in$ $[0,1] \mapsto R \in \mathbb{R}^{2}$ 的逆映射 $R \in \mathbb{R}^{2} \mapsto t \in[0,1]$ 的公式. 影响参数值 $t$ 的量既有 曲线段的权因子，又有由曲线段的控制顶点和此点所决定的三角形面积等几何 量. 把 (24) 式与 (4) 式中的第 3 式做比较, 不难发现有理三次圆雉曲线段上的参 数表达式与有理二次圆雉曲线段上的参数表达式极其相似, 它们的差别仅在于 其分母式的第二项中，量值 $\sqrt{\Delta_{0} / \Delta_{2}}$ 的因子是 $\sqrt{k \cdot \omega_{3} / \omega_{0}}$ 还是 $\sqrt{\omega_{2} / \omega_{0}}$, 因此只 要对于有理三次 Bézier 曲线所表示的椭圆弧、双曲线弧和抛物线弧, 分别计算出 相应的量值 $k$, 就能根据定理 4 , 相应地得到各类有理三次圆雉曲线段上参数的表 达式.

下面分别讨论曲线为椭圆弧、双曲线弧和抛物线弧时其参数的几何意义. 
首先考虑有理三次椭圆弧, 如 2.2 节建立坐标系, 则当边向量 $R_{0} R_{1}$ 与 $R_{2} R_{3}$ 不平行时, 必有

$$
Q_{1}=\left(\begin{array}{c}
a \cos \xi-\left.\frac{a \lambda_{0}}{2 \lambda_{1}} \cdot \frac{d \varphi}{d u}\right|_{u=0} \cdot \sin \xi \\
b \sin \xi+\left.\frac{b \lambda_{0}}{2 \lambda_{1}} \cdot \frac{d \varphi}{d u}\right|_{u=0} \cdot \cos \xi
\end{array}\right)=\left(\begin{array}{c}
a \cos \eta+\left.\frac{a \lambda_{2}}{2 \lambda_{1}} \cdot \frac{d \varphi}{d u}\right|_{u=1} \cdot \sin \eta \\
b \sin \eta-\left.\frac{b \lambda_{2}}{2 \lambda_{1}} \cdot \frac{d \varphi}{d u}\right|_{u=1} \cdot \cos \eta
\end{array}\right) .
$$

把上式看作以 $\left.\frac{\lambda_{0}}{2 \lambda_{1}} \cdot \frac{d \varphi}{d u}\right|_{u=0},\left.\frac{\lambda_{2}}{2 \lambda_{1}} \cdot \frac{d \varphi}{d u}\right|_{u=1}$ 为未知量的二元一次方程组, 求解得到

$$
\left.\frac{\lambda_{0}}{2 \lambda_{1}} \cdot \frac{d \varphi}{d u}\right|_{u=0}=\left.\frac{\lambda_{2}}{2 \lambda_{1}} \cdot \frac{d \varphi}{d u}\right|_{u=1}=\tan \frac{\eta-\xi}{2} .
$$

将 (9) 式中的参数 $t$ 看成 $u$, 可知

$$
\left.\frac{d \varphi}{d u}\right|_{u=0}=2 \sqrt{\frac{\lambda_{2}}{\lambda_{0}}} \cdot \sin \frac{\eta-\xi}{2},\left.\quad \frac{d \varphi}{d u}\right|_{u=1}=2 \sqrt{\frac{\lambda_{0}}{\lambda_{2}}} \cdot \sin \frac{\eta-\xi}{2} .
$$

将上式代入 (29) 式, 可得

$$
\frac{\lambda_{1}^{2}}{\lambda_{0} \lambda_{2}}=\cos ^{2} \frac{\eta-\xi}{2}
$$

于是应用 (22) 式有

$$
\left(-\omega_{0}+3 \omega_{1} k\right)\left(-\omega_{3} k^{3}+3 \omega_{2} k^{2}\right)=4 \cos ^{2} \frac{\eta-\xi}{2} \omega_{0} \omega_{3} k^{3} .
$$

整理上式，可得关于 $k$ 的一元二次方程

$$
\omega_{1} \omega_{3} k^{2}-\left[3 \omega_{1} \omega_{2}+\frac{1}{3}\left(1-4 \cos ^{2} \frac{\eta-\xi}{2}\right) \omega_{0} \omega_{3}\right] k+\omega_{0} \omega_{2}=0,
$$

由此解出

$$
k_{1,2}=\frac{3 \omega_{2}}{2 \omega_{3}}+\frac{\omega_{0}}{6 \omega_{1}}\left(1-4 \cos ^{2} \frac{\eta-\xi}{2}\right) \pm \frac{1}{6} \sqrt{\delta}
$$

其中

$$
\delta=\left[\frac{9 \omega_{2}}{\omega_{3}}-\frac{\omega_{0}}{\omega_{1}}\left(1-2 \cos \frac{\eta-\xi}{2}\right)^{2}\right]\left[\frac{9 \omega_{2}}{\omega_{3}}-\frac{\omega_{0}}{\omega_{1}}\left(1+2 \cos \frac{\eta-\xi}{2}\right)^{2}\right] .
$$

由此可知, 当边向量 $R_{0} R_{1}$ 与 $R_{2} R_{3}$ 不平行时, 有理三次椭圆弧上一点 $P$ 的参数 为

$$
t=t(\varphi)=\frac{\sin \frac{\varphi-\xi}{2}}{\sin \frac{\varphi-\xi}{2}+\sin \frac{\eta-\varphi}{2} \sqrt{k \cdot \frac{\omega_{3}}{\omega_{0}}}}
$$

其中实数 $k$ 如 (31) 式所示.

当边向量 $R_{0} R_{1}$ 与 $R_{2} R_{3}$ 平行时, 我们有 $S_{0}=S_{1}, S_{2}=S_{3}$. 将这两等式代 入 (19) 式, 得到 $k=\omega_{0} / 3 \omega_{1}=3 \omega_{2} / \omega_{3}$. 这种情形恰巧是 (31) 式中取 $\eta-\xi$ 为 $\pi$ 的 特殊情形, 因此点 $P$ 的参数表达式 (33) 仍然适合边向量 $R_{0} R_{1}$ 与 $R_{2} R_{3}$ 平行的情 形. 
类似地, 对有理三次双曲线弧, 如 2.2 节建立坐标系, 则可求得有理三次双 曲线弧上一点 $P$ 的参数为

$$
t=t(\varphi)=\frac{\sin \frac{\varphi-\xi}{2}}{\sin \frac{\varphi-\xi}{2}+\sin \frac{\eta-\varphi}{2} \sqrt{k \cdot \frac{\omega_{3}}{\omega_{0}} \cdot \frac{\cos \xi}{\cos \eta}}} .
$$

其中

$$
k_{1,2}=\frac{3 \omega_{2}}{2 \omega_{3}}+\frac{\omega_{0}}{6 \omega_{1}}\left(1-\frac{4 \cos ^{2} \frac{\xi+\eta}{2}}{\cos \xi \cos \eta}\right) \pm \frac{1}{6} \sqrt{\delta},
$$

且

$$
\delta=\left[\frac{9 \omega_{2}}{\omega_{3}}-\frac{\omega_{0}}{\omega_{1}}\left(1-\frac{2 \cos \frac{\xi+\eta}{2}}{\sqrt{\cos \xi \cos \eta}}\right)^{2}\right]\left[\frac{9 \omega_{2}}{\omega_{3}}-\frac{\omega_{0}}{\omega_{1}}\left(1+\frac{2 \cos \frac{\xi+\eta}{2}}{\sqrt{\cos \xi \cos \eta}}\right)^{2}\right] .
$$

最后, 对有理三次抛物线弧, 如 2.2 节建立坐标系, 则可求得有理三次抛物 线上一点 $P$ 的参数为

$$
t=t(\varphi)=\frac{\sin \frac{\varphi-\xi}{2} \sin \frac{\eta}{2}}{\sin \frac{\varphi-\xi}{2} \sin \frac{\eta}{2}+\sin \frac{\eta-\varphi}{2} \sin \frac{\xi}{2} \sqrt{k \cdot \frac{\omega_{3}}{\omega_{0}}}}
$$

其中

$$
k_{1,2}=\frac{3 \omega_{2}}{2 \omega_{3}}-\frac{\omega_{0}}{2 \omega_{1}} \pm \frac{1}{2} \sqrt{\left(\frac{9 \omega_{2}}{\omega_{3}}-\frac{\omega_{0}}{\omega_{1}}\right)\left(\frac{\omega_{2}}{\omega_{3}}-\frac{\omega_{0}}{\omega_{1}}\right)} .
$$

定理 6 用有理三次 Bézier 曲线 (18) 表示由 (7)、(10)、(13) 式所给出的椭 圆弧、双曲线弧和抛物线弧时, 曲线上任一点 $P$ 所对应的参数的几何意义分别 如 (33)、(34)、(37) 式所示.

由定理 6 可知，表达式 (33)、(34)、(37) 对有理三次椭圆弧、双曲线弧和抛 物线弧分别给出了方便简洁的反求参数值的方法, 因此利用这些公式可以在任 意指定点处对有理三次圆雉曲线段进行分割. 把这些公式与定理 4 中的公式相 比较, 容易看出两者之间惊人地相似, 它们的差别仅在于分母的第 2 项中的系数 $k$ 是否等于 1 ; 不但如此, 以上表达式还表明, 若想改变曲线段的参数化状况, 只需在保持 $\omega_{1}^{2} /\left(\omega_{0} \omega_{2}\right), \omega_{2}^{2} /\left(\omega_{1} \omega_{3}\right)$ 之值的条件下, 通过调整权因子来改变系数 $K=k \omega_{3} / \omega_{0}$ 的值. 当 $K$ 的值增大 (减小) 时, $P$ 点的参数值 $t$ 将随之减小 (增大). 而由 (19) 式可知, 比值 $\omega_{i} / \omega_{i+1}(i=0,1,2)$ 与 $k$ 的值成正比, 因此我们得到

推论 2 对有理三次 Bézier 曲线 (18) 所表示的圆雉曲线段, 若保持 $\omega_{1}^{2} /\left(\omega_{0} \omega_{2}\right)$, $\omega_{2}^{2} /\left(\omega_{1} \omega_{3}\right)$ 之值不变, 但调整权因子的比值 $\omega_{i+1} / \omega_{i}(i(\bmod 4))$, 则当此比值增大 (减小) 时, 曲线上 $P$ 点的原参数值 $t$ 将随之增大 (减小).

推论 2 为有理三次圆雉曲线段的重新参数化和最优参数化提供了理论基 础. 


\section{参考文献}

1 Faux I D, Pratt M J. Computational Geometry for Design and Manufacture. Chichester: Ellis Horwood Limited, 1979

2 Forrest A R. The twisted cubic curves: a computer aided geometric design approach. Computer-Aided Design, 1980, 12(4): 165 172

3 Farin G. Algorithms for rational Bézier curves. Computer-Aided Design, 1983, 15(2): 73 77

4 Pigel L. Representation of quadric primitives by rational polynomials. Computer Aided Geometric Design, 1985, 2(1-3): 151 155

5 Lee E T Y. The rational Bézier representation for conic. In: Farin G E, ed. Geometric Modeling: Algorithms and New Trends. Philadelphia: SIAM, 1987. 3 19

6 Pigel L, Tiller W. A menagerie of rational B-spline circles. IEEE Computer Graphics and Application, 1989, (9): 48 56

7 Wang Guojin. Rational cubic circular arcs and their application in CAD. Computers in Industry, 1991, 16(3): $283 \sim 288$

8 Wang Guojin, Wang Guozhao. The rational cubic Bézier representation of conics. Computer Aided Geometric Design, 1992, 9(6): 447 455

9 Farin G. Curves and Surfaces for Computer Aided Geometric Design, A Practical Guide. 3rd ed. New York: Academic Press, 1993

10 施法中. 计算机辅助几何设计与非均匀有理 B 样条 (CAGD \& NURBS). 北京: 北京航空航天大学出 版社, 1994

11 施法中. 论采用有理 Bézier 表示的圆弧曲线的参数化. 见: 张彩明, 方渏, 编. 第一届全国几何设 计与计算学术会议论文集. 东营: 石油大学出版社, 2002. 60 69 